\title{
Alimentos funcionales, alfalfa y fitoestrógenos
}

\author{
Alejandro De Jesús Cortés-Sánchez, ${ }^{1}$ José Rubén León-Sánchez, ${ }^{2}$ \\ Francisco Javier Jiménez-González, ${ }^{2}$ Mayra Díaz-Ramírez, ${ }^{3}$ \\ Adriana Villanueva-Carvajal, ${ }^{4}$ Cynthia A. Guzmán-Medina ${ }^{5}$
}

\begin{abstract}
'Secretaría de Salud. Comisión Federal para la Protección contra Riesgos Sanitarios. Departamento de Microbiología, Tlalpan, Ciudad de México, México. *Autor de correspondencia: alecortes_1@hotmail.com

${ }^{2}$ Facultad de Medicina UAEMex, Av. Paseo Tollocan esq. Jesús Carranza № 134 502, residencial Colón y Col. Cipres. C.P. 50120 Toluca de Lerdo, Estado de México, México.

${ }^{3}$ Departamento de Alimentos. División de Ciencias Biológicas y de la Salud. Universidad Autónoma Metropolitana (UAM), Unidad Lerma. Estado de México, México.

${ }^{4}$ Facultad de Ciencias Agrícolas, Universidad Autónoma del Estado de México, Campus Universitario "El Cerrillo" A.P. 435, Toluca, Estado de México C.P. 50200, México.

${ }^{5}$ Secretaría de Salud. Comisión Federal para la Protección contra Riesgos Sanitarios. Control Analítico, Tlalpan, Ciudad de México, México.
\end{abstract}

\section{Functionals foods, alfalfa and phytoestrogens}

\begin{abstract} in its consumption.

Editor: Hernández Fernández, J.

Citation: Cortés, A., León, J., Jiménez, F., Díaz, M., Villanueva, A \& Guzmán, C. (2016). Alimentos funcionales, alfalfa y fitoestrógenos. Revista Mutis 6(1), 2840, doi: http://dx.doi.org/10.21789/22561498.1110
\end{abstract}

Functional food development has been increased during the last years due to the population interest in foods that have not only a nutritional function but a health benefit as well reducing the risk of suffering several diseases. Alfalfa has been intended as cattle feed, although several investigations have reported that alfalfa contains bioactive compounds - phytochemicals- proteins with an estrogenic effect, and antimicrobial and antioxidant compounds, being classified as a functional food with protection against cancer, diabetes and cardiovascular and other diseases. The aim of the present study is to offer a general information outline, through a bibliographic review, of functional foods' status, focusing on alfalfa and its phytochemicals (phytoestrogens), compounds that give it its functional food character and its adverse effects shown in the reproductive system of experimental animals as well. Such dichotomy between adverse and beneficial effects is the sign that shows that it is needed more information and more studies to be done in order to establish the effect of phytoestrogens in the development and reproductive function in animals, its agonistic and antagonistic effect and risk-benefit balance

Received: February 23, 2016; Accepted: March 31, 2016; Published on line: May 31, 2016.

Copyright: @2016 Cortés, A., León, J., Jiménez, F., Díaz, M., Villanueva, A \& Guzmán, C. This is an open-access article, which permits unrestricted use, distributions and reproduction in any medium, provided the original author and source are credited.

Competing Interests: The authors have no conflict of interest.
Keywords: foods, bioactive, phytochemicals, coumestrol, phytoestrogens.

\section{RESUMEN}

El desarrollo de alimentos funcionales en los últimos años ha ido en aumento debido principalmente, al interés de la población por alimentos que no solo cumplan con la función nutricional sino también por que tengan un efecto benéfico en salud reduciendo así el riesgo a padecer diversas enfermedades. La alfalfa ha sido generalmente un alimento destinado para la ali- 
mentación del ganado, sin embargo, en diversas investigaciones alrededor del mundo se ha reportado que contiene compuestos bioactivos -fitoquímicosy proteínas con efecto estrogénico, antimicrobiano y antioxidante, así como también protector contra enfermedades cardiovasculares, cáncer y diabetes, entre otras, clasificándola potencialmente como un alimento funcional. El presente documento tiene como objetivo dar un bosquejo general informativo a través de la búsqueda y revisión bibliográfica acerca de los alimentos funcionales y en especial de la alfalfa y constituyentes fitoquímicos (fitoestrógenos) que la ubican dentro de este grupo de alimentos; así como los hallazgos que se han reportado como efectos adversos en animales experimentales específicamente en el aparato reproductivo debido al consumo de este tipo de fitocompuestos. Esta dicotomía entre los efectos adversos y benéficos no es más que la señalización de que aún falta mucha más información y estudios que realizar respecto al resultado que pueden tener los fitoestrógenos en el desarrollo y función reproductiva de animales, el agonismo y antagonismo de su efecto así como la concordancia en el riesgo-beneficio de su consumo.

Palabras clave: alimentos, bioactivo, fitoquímicos, coumestrol, fitoestrógenos.

\section{INTRODUCCIÓN}

En la actualidad la elaboración, autosuficiencia y disponibilidad de alimentos se destaca por ser un tema de gran importancia, ya que una nutrición adecuada, requiere de seguir una dieta que sea suficiente y equilibrada que incluya los nutrientes básicos que recomiendan los expertos (Martínez y Villezca, 2015).

El acelerado estilo de vida actual ha generado cambios en la alimentación a nivel mundial. Los nuevos y algunas veces poco saludables hábitos alimenticios de la población junto con factores como el sedentarismo y estrés, favorecen el incremento de enfermedades como diabetes, obesidad, hipertensión arterial y cáncer, entre otras, generándose así un problema de salud pública alrededor del mundo. Por otra parte, en países en desarrollo las desigualdades económicas hacen que gran parte de la población no acceda a los alimentos de calidad o en cantidad suficiente, ocasionando episodios de desnutrición y retraso en el desarrollo físico (Rubiano, 2006).
Otro punto importante en el ámbito de los alimentos es la inocuidad, que de acuerdo a la Organización Mundial de la Salud (OMS), es la nulidad para generar efectos o daños nocivos a la salud humana por parte de los alimentos al ser consumidos. Para tal fin se incluyen políticas y estrategias que engloban en sí toda la cadena alimenticia desde su manufactura hasta su consumo.Así, la inocuidad debe ser el común denominador; es decir, desde los productores primarios, sean del campo o del mar, hasta que el alimento llega a la mesa del consumidor, donde se requiere observar prácticas en cada etapa del proceso que aseguren la inocuidad de los alimentos (OMS, octubre, 2009; Badui, 2015).

Como consecuencia de la falta de inocuidad en los alimentos, se presentan las llamadas enfermedades transmitidas por alimentos (ETA), las cuales tienen impacto social y económico por los gastos médicos y hospitalarios involucrados, por el ausentismo laboral y bajo rendimiento en el trabajo que afecta la productividad de las empresas, y por el potencial daño permanente como secuela de la enfermedad y por muerte las cuales se estiman en $\mathbf{2 0 0}$ millones de personas al año en el mundo. Desde hace ya varios años la inocuidad se ha considerado un tema relevante para los consumidores y en consecuencia, para la industria alimentaria mundial; para lo cual cada país cuenta con requerimientos oficiales dirigidos a la industria de alimentos a fin de garantizar la inocuidad alimentaria. Para asegurar la inocuidad, la industria alimentaria alrededor del mundo dispone de diversos sistemas internacionalmente reconocidos, como son: Safe Quality Food (SQF), International Food Standard (IFS), British Retail Consortium (BRC), Food Safety System Certification (FSSC), American Institute of Baking (AIB) y Grocery Manufacturers Association (GMA-safe). Todos reconocidos por el Global Food Safety Iniciative (GFSI) y fundados en buena parte en la implementación del Hazard Analysis and Critical Control Points (HACCP), que a su vez tiene base en el cumplimiento de las buenas prácticas de manufactura o requisitos y los procedimientos operativos de estandarización de saneamiento. En México de manera general, las preocupaciones del público consumidor sobre la inocuidad están relacionadas con la presencia de plaguicidas y de aditivos alimentarios (especialmente conservadores y colorantes), mientras que las autoridades sanitarias presentan un énfasis y cuidado por contaminaciones 
con agentes microbiológicos y químicos. Así, para realizar acciones que conduzcan a la inocuidad en toda la cadena alimentaria se requiere de muchos controles y normatividades por diferentes organismos, tales como la Secretaría de Agricultura, Ganadería, Desarrollo Social, Pesca y Alimentación (SAGARPA), Servicio Nacional de Sanidad, Inocuidad y Calidad Agroalimentaria (SENASICA), Dirección General de Inocuidad Agroalimentaria, Avícola y Pesquera (DGIAAP), Comisión Federal para la Protección contra Riesgos Sanitarios (COFEPRIS) y Procuraduría Federal del Consumidor (PROFECO); donde cada entidad solicita determinados requerimientos que la cadena alimentaria debe cumplir (Badui, 2015).

Por otra parte, a nivel internacional además del tema de la inocuidad alimentaria, el interés desde hace ya varios años se ha ido inclinando a la relación entre alimentación y salud, y los objetivos sanitarios a los que se dirigen los alimentos funcionales están enfocados a diferentes enfermedades de considerable valor social y económico buscando así soluciones a los problemas de desnutrición, falta de desarrollo físico, aumento en la incidencia de enfermedades crónicas no trasmisibles (ECNT) como diabetes, hipertensión arterial, obesidad, sobrepeso, síndrome metabólico e hipercolesterolemia, osteoporosis, cáncer (estrés oxidativo), y el crecimiento y desarrollo en etapas periconcepcional, fetal, perinatal, adolescencia, embarazo, así como a los efectos de la nutrición sobre las funciones cognitivas, inmunitarias, capacidad de trabajo, crecimiento y composición corporal y rendimiento deportivo. Estudios científicos y desarrollos tecnológicos pretenden fomentar el consumo de alimentos que además de una nutrición básica aporten beneficios adicionales para la salud y bienestar de la población, teniendo en cuenta sus características genéticas, ambientales, sociales y culturales. De tal manera, que en el futuro los alimentos puedan no solo permitir un crecimiento y desarrollo óptimo desde la gestación y en todas las etapas de la vida, sino que también puedan potenciar las capacidades físicas y mentales del individuo, y disminuir el riesgo a padecer enfermedades. Además, la población general está cada vez más consciente de su autocuidado y busca en el mercado productos que contribuyan a su salud y bienestar (Araya y Lutz, 2003; Rubiano, 2006; Olagnero et al., 2007; Ortega et al., 2010).
En diversos países se han desarrollado estrategias que están encaminadas a reducir el riesgo de desarrollar enfermedades crónicas no transmisibles (ECNT) de la población, a través de promover en la dieta el consumo de alimentos, que contengan, por ejemplo, fuentes de fibra dietética como legumbres, cereales integrales, vegetales y frutas; pues estas están asociadas estrechamente con la dieta alimenticia (Alvídrez et al., 2002; Bolet y Socarrás, 2010).

El estudio de la relación entre alimentación y salud, tiende a ser una acción más preventiva que correctiva de déficits nutricionales, esto porque hay investigaciones que asocian el consumo de alimentos vegetales (frutas, verduras, cereales y leguminosas) con efectos protectores contra enfermedades crónico degenerativas, el cáncer y enfermedades cardiovasculares. Dicha correspondencia se encuentra sustentada a su vez por estudios epidemiológicos in vivo, in vitro y clínicos que muestran la relación inversa entre la presencia de estas enfermedades y el consumo de vegetales en la dieta (Araya y Lutz, 2003; Chasquibol et al., 2003).

En la presente revisión se aborda de manera general e informativa el tema de los alimentos funcionales, las razones por las que han ido adquiriendo relevancia en los últimos años en el área de salud y ciencia de los alimentos tanto para la sociedad como para la investigación, así como los compuestos químicos presentes y su modo de acción, principalmente los provenientes de las leguminosas. Dentro de este contexto se aborda el caso de la alfalfa ya que debido a su naturaleza, composición y efectos biológicos, hoy en día se le categoriza potencialmente como un alimento funcional. Así mismo, también se aborda el probable efecto antagonista reportado por diversos investigadores de los fitoquímicos -fitoestrogenos-que contienen la alfalfa y otros vegetales, en animales.

\section{Alimentos funcionales}

En los países industrializados el concepto de nutrición ha ido cambiando, desde el significado básico del aporte de nutrientes necesarios para que un organismo desarrolle sus funciones, hasta la concepción actual que considera a los alimentos como promotores de la salud (Lorente y Serra, 2001). 
Los alimentos funcionales se definen como los productos alimenticios de origen animal o vegetal, consumidos en la dieta diaria, que además de aportar nutrientes poseen componentes bioactivos que ejercen efectos fisiológicos o psicológicos más a allá de su valor nutricional que resultan benéficos para la salud, reduciendo el riesgo de contraer alguna enfermedad (Lorente y Serra, 2001; Araya y Lutz, 2003; Serrano et al., 2006).

Los alimentos funcionales tienen su origen en el interés creciente de la población por la relación entre la alimentación y la salud, el envejecimiento progresivo, aumento de enfermedades atópicas, preocupación por reducir costos sanitarios, relevancia del etiquetado nutricional, prevención de enfermedades y desarrollo tecnológico. Además, factores como el estilo de vida, hábitos alimentarios, edad, sexo, estado físico y de salud generan distintos grupos poblacionales y estos demandan diferentes necesidades de alimento, por lo que la diversificación de los alimentos funcionales puede aplicarse en función de cubrir las necesidades de los distintos grupos. Cabe señalar que el efecto de un alimento funcional será significativo o nulo en función de la dieta o los hábitos alimenticios de la población a quien se dirige. Algunos grupos que pueden potencialmente beneficiarse de los alimentos funcionales son: a) Grupos con necesidades especiales tales como mujeres embarazadas, posmenopáusicas, en etapa fértil, niños en crecimiento, fumadores y personas de la tercera edad. b) Personas con dietas inapropiadas o poco saludables, mujeres con dietas restrictivas para perder peso, deportistas de alto rendimiento. c) Personas bajo tratamientos farmacológicos que interfieren en la absorción de nutrientes, consumo frecuente de laxantes, personas con padecimientos en el aparatos gastrointestinal (enfermedad de Crohn, intolerancia a la lactosa, síndrome de intestino corto) d) Vegetarianos. Se debe eliminar la idea de que estos alimentos cambiarán por arte de magia los errores dietéticos tanto en exceso como por defecto, fortaleciendo la necesidad de llevar una dieta equilibrada y variada así como la práctica regular de actividad física (Varela, 2010).

El fomento de la salud es un tema de gran consideración para todos los individuos y profesionales sanitarios, que a su vez favorece la investigación y desarrollo en el campo de los alimentos funcionales los cuales se han llegado a clasificar en base al beneficio en la salud al que se asocian o patologías que pueden prevenir o controlar (Ortega et al., 2010). Así, algunos alimentos y en específico los de origen vegetal, son altamente apreciados por su potencial terapéutico atribuido a su contenido de algunos componentes provenientes del metabolismo secundario de las plantas y conocidos como fitoquímicos bioactivos (polifenoles, fenoles, isoprenoides, ácidos grasos esenciales, terpenos, organosulfurados, $\beta$-glucanos) (Serrano et al., 2006; Pérez y Ávalos, 2009). Se han descrito 11 categorías de ingredientes con actividad fisiológica presentes en gran variedad de alimentos, las cuales son: fibras alimentarias, oligosacáridos, alcoholes derivados de azúcares, ácidos grasos poliinsaturados, péptidos y proteínas, glucósidos, isoprenoides, vitaminas, alcoholes, fenoles, colinas (lecitina), bacterias del ácido láctico, y minerales, entre otros (Cortés et al., 2005).

Los alimentos son considerados un gran complejo químico y biológico, resultante de las interacciones de sus constituyentes naturales y operaciones industriales-culinarias que se emplean para su consumo, dando lugar a su vez a cambios profundos en las propiedades fisicoquímicas del alimento, determinando en buena parte la biodisponibilidad de sus componentes y su función en el metabolismo intermediario. Lo anterior, ha representado una oportunidad para la industria alimentaria, de generar nuevas líneas de productos, con valor agregado y de afinidad con los consumidores (Araya y Lutz, 2003; Cortés et al., 2005).

Se han desarrollado diversos procedimientos para producir alimentos funcionales que consisten en:

1. Incrementar la concentración de un componente natural del alimento para alcanzar una concentración que se espera que induzca los efectos deseados, por ejemplo, la fortificación con micronutrientes para lograr una ingesta mayor que las recomendaciones dietéticas, compatible con los valores sugeridos para disminución de riesgos de enfermedades.

2. Agregar un componente que no está normalmente presente en la mayor parte de los alimentos.

3. Reemplazar un componente del alimento, generalmente un macronutriente cuya ingesta 
sea excesiva y que muestre efectos nocivos, por ejemplo el reemplazo de grasa por fibra dietética, componente beneficioso para la salud. Hay que mencionar que el advenimiento de la ingeniería genética, así como técnicas en cultivo y cría, incorporación a granel e ingeniería de matrices por impregnación a vacío (IV) descrito a través de la acción del mecanismo hidrodinámico (HDM) transporte de materia en un sistema sólido porosolíquido, han dado más opciones para la fabricación de alimentos funcionales (Araya y Lutz, 2003; Cortés et al., 2005).

Así mismo, hay que considerar que existen fases en el desarrollo de alimentos funcionales como son: la selección y definición de compuestos fisiológicamente activos, el desarrollo de técnicas para identificar y valorar la actividad de dichos ingredientes en materia prima y producto terminado, el estudio de propiedades físicas, químicas y biológicas del alimento, el análisis de los procesos de absorción y de metabolización del ingrediente con actividad fisiológica por el organismo, el estudio mediante procedimientos acelerados de la estabilidad del constituyente activo en la fórmula final en distintas condiciones, la valoración de efectos beneficiosos en un modelo animal preparándose para los ensayos clínicos, los estudios de toxicidad aguda y crónica en modelos animales, y el establecimiento de dosis mínimas, máximas y experimentación clínica siguiendo protocolos científicos adecuados en adultos, niños sanos, así como en enfermos y personas mayores (si en ellos tuviera indicación el principio activo)(Cortés et al., 2005).

Existen diversos alimentos funcionales los cuales incluyen una gran variedad de ingredientes alimenticios clasificados por su función, en probióticos, prebióticos, simbióticos, proteínas o péptidos, lípidos, minerales, vitaminas, fenoles o polifenoles y compuestos azufrados (tabla 1).

Tabla 1. Ingredientes funcionales o componentes bioactivos presentes en los alimentos, definición y beneficios a la salud.

\begin{tabular}{|c|c|c|c|}
\hline $\begin{array}{l}\text { Ingrediente } \\
\text { funcional }\end{array}$ & Definición o característica & Ejemplos & Beneficios a la salud \\
\hline Probiótico & $\begin{array}{l}\text { Microorganismos vivos } \\
\text { específicamente bacterias, que } \\
\text { administrados en dosis adecuadas, no } \\
\text { causan efectos patológicos y confieren } \\
\text { beneficios en salud al hospedero. }\end{array}$ & $\begin{array}{c}\text { B. bifidum } \\
\text { Saccharomyces boulardii } \\
\text { L. acidophilus } \\
\text { L. casei } \\
\text { Propionibacterium } \\
\text { freudenreichii }\end{array}$ & $\begin{array}{l}\text { Reducción de intolerancia a la lactosa, } \\
\text { diarreas infecciosas, } \\
\text { estimulaciónde sistema inmunológico }\end{array}$ \\
\hline Prebiótico & $\begin{array}{l}\text { Ingredientes resistentes a la digestión } \\
\text { intestinal con capacidad fermentable } \\
\text { que favorecen el crecimiento de } \\
\text { bacterias y dinamismo colónico } \\
\text { con efectos benéficos para el } \\
\text { organismo huésped. }\end{array}$ & $\begin{array}{c}\text { Galacto-oligosacaridos, } \\
\text { ácido lactobiónico, inulina y } \\
\text { fructooligosacáridos (FOS), lignina, } \\
\text { celulosa, pectinas, } \\
\beta \text {-glucano }\end{array}$ & $\begin{array}{l}\text { Regulación y salud intestinal } \\
\text { Reducción de riesgos hacia } \\
\text { estreñimiento, diarreas infecciosas, } \\
\text { osteoporosis aterosclerosis y } \\
\text { enfermedad cardiovascular obesidad, } \\
\text { diabetes mellitus tipo 2, } \\
\text { hipocolesterolemico e hipolipemiante }\end{array}$ \\
\hline Simbiótico & $\begin{array}{l}\text { Mezcla de uno o más probióticos con } \\
\text { uno o varios compuestos prebióticos. }\end{array}$ & $\begin{array}{c}\text { Lactobacillus casei } \\
\text { Sancor CRL 431+ } \\
\text { Lactobacillus } \\
\text { acidophilus Sancor } \\
\text { CRL } 730 \text { (johnsonii) } \\
\text { + Fructanos } \\
\text { naturales (Inulina-FOS). } \\
\text { Bifidobacterium } \\
\text { animalis } \\
\text { DN } 173010 \\
\text { + Prebiótico } \\
\text { FOS (inulina). } \\
\end{array}$ & $\begin{array}{l}\text { Favorecer el desarrollo/actividad de } \\
\text { los probióticos para potenciar sus } \\
\text { propiedades saludables, generando un } \\
\text { efecto sinérgico. Esto implica que un } \\
\text { producto solo puede ser denominado } \\
\text { simbiótico si demuestra favorecer un } \\
\text { efecto beneficioso mayor al de la suma } \\
\text { de los generados, separadamente, por } \\
\text { sus integrantes. }\end{array}$ \\
\hline
\end{tabular}




\begin{tabular}{|c|c|c|c|}
\hline $\begin{array}{l}\text { Proteínas o } \\
\text { péptidos }\end{array}$ & $\begin{array}{c}\text { Compuestos poliméricos formados } \\
\text { por aminoácidos unidos a través de } \\
\text { enlaces peptídicos. }\end{array}$ & $\begin{array}{l}\text { Casokininas, aS1-caseína, b-caseína, } \\
\text { K-caseina, lactokininas, lactoferrinas. }\end{array}$ & $\begin{array}{l}\text { Inmunomoduladores, } \\
\text { antimicrobianos, } \\
\text { y antihipertensivos. }\end{array}$ \\
\hline Lípidos & $\begin{array}{l}\text { Moléculas generalmente hidrofóbicas } \\
\text { constituidas por cadenas alifáticas } \\
\text { saturadas o insaturadas, lineales o } \\
\text { aromáticas. Además de contener } \\
\text { carbono, hidrogeno, oxigeno, fosforo, } \\
\text { azufre y nitrógeno, entre otros. }\end{array}$ & $\begin{array}{c}\text { Acido butírico, ácido linoleico, ácido } \\
\text { linoleico conjugado, fosfolípidos, ácido } \\
\text { eicosapentaenoico, (20:5n-3) } \\
\text { y el DHA docosahexaenoico, (22:6n-3), } \\
\text { terpenos, esteroides, isoprenoides } \\
\text { mixtos, esteroles. }\end{array}$ & $\begin{array}{c}\text { Reducción de riesgo de enfermedades } \\
\text { cardiovasculares, } \\
\text { hipocolesterolemico, } \\
\text { antiinflamatorio, } \\
\text { antitromboticos. }\end{array}$ \\
\hline Minerales & $\begin{array}{l}\text { Elemento químico inorgánico de } \\
\text { composición definida. }\end{array}$ & $\mathrm{Ca}, \mathrm{Fe}, \mathrm{Mg}$, Se. & $\begin{array}{c}\text { Prevención y control de osteoporosis, } \\
\text { anemia ferropénica, protector frente } \\
\text { aenfermedades cardiovasculares y } \\
\text { degenerativas. }\end{array}$ \\
\hline Vitaminas & $\begin{array}{l}\text { Sustancias orgánicas, algunas } \\
\text { esenciales para la nutrición y } \\
\text { mantenimiento de funciones } \\
\text { metabólicas en la célula. }\end{array}$ & A, D, E, K, y C. & $\begin{array}{c}\text { Antioxidantes, prevención y control de } \\
\text { osteoporosis, disminución del riesgo } \\
\text { enfermedad cardiovascular, mejora } \\
\text { de la salud cutánea y retraso del } \\
\text { envejecimiento de lapiel. } \\
\end{array}$ \\
\hline $\begin{array}{l}\text { Fenoles o } \\
\text { Polifenoles }\end{array}$ & $\begin{array}{l}\text { Conjunto heterogéneo de moléculas } \\
\text { con la similitud de compartir en } \\
\text { su estructura uno varios grupos } \\
\text { bencénicos sustituidos por funciones } \\
\text { hidroxílicas. }\end{array}$ & $\begin{array}{l}\text { Curcumina, ácido cafeico, ferúlico,y } \\
\text { clorogénico, flavonas, flava nonas, } \\
\text { catequinas, antocianinas, isoflavonas, } \\
\text { coumestanos y lignanos. }\end{array}$ & $\begin{array}{l}\text { Reducen el riesgo de enfermedades } \\
\text { cardiovasculares,neoplasias, } \\
\text { antioxidantes, antimicrobianos, } \\
\text { estrogenicos. }\end{array}$ \\
\hline $\begin{array}{l}\text { Compuestos } \\
\text { azufrados }\end{array}$ & $\begin{array}{l}\text { Compuestos organosulfurados } \\
\text { solubles e insolubles en agua, algunos } \\
\text { además contienen nitrógeno. }\end{array}$ & $\begin{array}{c}\text { S-alilcisteína, } \\
\text { S-alilmercaptocisteína, } \\
\text { sulfuro de dialilo, } \\
\text { disulfuro de dialilo, trisulfuro de } \\
\text { dialilo. }\end{array}$ & $\begin{array}{c}\text { Antioxidante, } \\
\text { reducción de LDL sanguíneos, } \\
\text { anti-inflamatorio, antiatrogénico, } \\
\text { antitrombótico relacionado } \\
\text { a su capacidad de inhibir } \\
\text { la síntesis de prostanoides. } \\
\end{array}$ \\
\hline
\end{tabular}

Los alimentos funcionales son un mercado en aumento que mueve al año alrededor del mundo aproximadamente 100.000 millones de euros (Murcia, 2013). Estadísticas de países desarrollados registran un creciente y acelerado consumo de productos alimenticios que ofrezcan beneficios para la salud, siendo esto por tanto un indicador del interés que existe por mejorar la nutrición y la salud de la población (Madrigal y Sangronis, 2007). Tan solo en los Estados Unidos se estima por este tipo de alimentos una facturación cercana a los 40.000 millones de euros, y en países como Japón y miembros de la Unión Europea se encuentran cada vez mejor posicionados, además se ha pronosticado una mayor expansión en los próximos años en países con potencial industrial como: China, India, Australia, Malasia o Corea del Sur, esto debido al aumento en incidencia de enfermedades cardiovasculares, además de problemas intestinales y alergias, que han popularizado el consumo de alimentos funcionales alrededor del mundo y que los consumidores los consideren, cada vez más a la hora de realizar las compras (Murcia, 2013). Aunado a lo anterior también continúa en incremento en el número de estudios científicos con el fin de demostrar los beneficios de los alimentos funcionales en la prevención de diversas enfermedades como las cardiovasculares y el cáncer (Madrigal y Sangronis, 2007).

El éxito de la industria alimentaria depende de la capacidad de adaptación e innovación de productos de calidad que satisfagan las expectativas y respondan a las necesidades sociales de los consumidores. La importancia de la innovación, transferencia y evolución, debe extenderse a la comunicación, búsqueda de información, de gobiernos, sociedades, y alianzas. Además, para conseguir una política de innovación, la empresa puede optar por el desarrollo interno de tecnología o bien por su transferencia. Los países líderes en investigación y desarrollo y por ende con los avances más importantes en el desarrollo de alimentos funcionales son los Estados Unidos, Japón, Alemania, Reino Unido y Francia (Cortés et al., 2005).

Los alimentos funcionales de origen vegetal representan una potencial fuente de componentes bioactivos (presentan actividad biológica en las células así como en los mecanismos fisiológicos con efectos benéficos para la salud) denominados fitoquímicos, siendo una amplia variedad de vegetales altamente apreciados 
por su potencial terapéutico atribuido al contenido de estos compuestos, los cuales son de sumo interés en la industria farmacéutica para el desarrollo de fármacos. Dentro de los fitoquímicos bioactivos en los alimentos están los fitoestrógenos, un grupo de compuestos no esteroidales que comprenden: isoflavonas (daidzeína, genisteína, biochanina A, gliciteina), coumestanos (coumestrol), lignanos (enterodiol y enterolactona) y flavonoides (naringenina), siendo las fuentes más abundantes las legumbres, frutas y leguminosas como trébol, alfalfa, soya, cascarilla de semilla de linaza y centeno (tabla 2) (Garrido et al., 2003; García y López, 2004; Serrano et al., 2006; Lenis et al., 2010; Cendales y Silva, 2011).

Los fitoestrógenos como las isoflavonas se encuentran en una variedad de vegetales, en forma de glicósidos (compuestos formados por la unión de uno o más carbohidratos con moléculas de diferente naturaleza química). Así, fitoquímicos como la genisteína, daidzeína y gliciteína en su forma glicosilada al ser ingeridas son hidrolizadas a agliconas (carbohidratos $u$ oligosacaridos que forma parte del glicósido), su forma activa por bacterias en el intestino delgado. Las agliconas son absorbidas por la mucosa intestinal y transportadas por la vena porta hasta el hígado, donde por acción de las enzimas glucoroniltransferasas y sulfotransferasas, forman conjugados de glucorónidos y sulfatos, siendo la primera la principal vía de biotransformación; posteriormente son excretados por la bilis y la orina. Sin embargo, estudios acerca de la biodisponibilidad de las isoflavonas mencionan que esta es mayor cuando se ingieren como ß-glicósidos que como agliconas. Por lo tanto, las etapas de biotransformación son de gran importancia en la acción de las isoflavonas (Garrido et al., 2003; Bonilla, 2004).

Tabla 2. Concentración de fitoestrógenos presente en diferentes alimentos en base húmeda.

\begin{tabular}{cc}
\hline Alimento & $\boldsymbol{\mu g} / \mathbf{1 0 0} \mathrm{g}$ \\
\hline Leche de soya & 2957.2 \\
\hline Frijol de soya & 103920 \\
\hline Tofu & 27150.1 \\
\hline Lentejas & 36.5 \\
\hline Alfalfa brote & 441.4 \\
\hline Ajo & 603.6 \\
\hline Brócoli & 94.1 \\
\hline Arándanos & 17.5 \\
\hline Semilla de girasol & 216.0
\end{tabular}

Se ha tratado de dilucidar el modo de acción de los fitoestrógenos en las plantas productoras, indicando que al parecer su función es protegerlas contra la fauna nociva, la radiación ultravioleta, actuando como captadoras de radicales libres potencialmente agresivos por su acción oxidante. Además, estos compuestos antioxidantes vegetales se han asociado con la regulación del crecimiento vegetal así como con la resistencia a determinadas infecciones bacterianas, víricas y micóticas (Lenis et al., 2010).

Entre las propiedades farmacológicas de los fitoestrógenos con aplicación en anímales se encuentra su actividad estrogénica (el típico estrógeno es el $17 \beta$-estradiol, que tiene funciones en la reproducción de vertebrados y es producido por ovarios, glándulas suprarrenales y placenta) (figura 1), la cual ha sido aplicada en terapias de reemplazo hormonal para el alivio de los síntomas asociados a la menopausia y osteoporosis en mujeres en etapa del climaterio (García y López, 2004; Serrano et al., 2006; Ortiz, 2010). Además, el consumo de fitoestrógenos en la dieta ha sido relacionado con efectos benéficos contra cáncer de próstata, obesidad, diabetes, inflamación, artritis, infarto miocárdico, neurodegeneración y cáncer de mama (García y López, 2004; Serrano et al., 2006).

Estos fitoquímicos de naturaleza fenólica son considerados protectores contra enfermedades cardiovasculares, al modificar el perfil lipídico, inhibiendo la oxidación, lipoproteínas de baja densidad (LDL) y triglicéridos favoreciendo el aumento de lipoproteínas de alta densidad (HDL) previniendo la ateroesclerosis. Estos compuestos además estimulan la actividad de la óxido nítrico sintetasa endotelial y producen la vasodilatación por la vía del óxido nítrico inhibiendo la formación de radicales libres, peróxido de hidrógeno y aniones superóxido teniendo por lo tanto actividad antioxidante y antitrombótica al inhibir la agregación plaquetaria (Cendales y Silva, 2011).

Respecto de los fitoquímicos aislados de leguminosas, se ha reportado que imitan la actividad del $17 \beta$-estradiol, pero sus efectos no son idénticos dado que pueden presentar actividad antiestrogénica. Este efecto se puede deber a que poseen grupos hidroxilos que consiguen alinearse en una posición estereoquímica similar a la del $17 \beta$ - estradiol y competir por el sitio activo del receptor de estrógeno endógeno en mamíferos (figura 1). De esta manera el balance entre la actividad estrogénica está determinado por la proporción 
del fitoestrógeno y estrógenos endógenos. Los compuestos estrogénicos de mayor importancia en las leguminosas son las isoflavonas y coumestanos; siendo la estrogenicidad de coumestanos $1 / 1000$ y de isoflavonas1/10 000 aproximadamente, en relación con la actividad del 17ß-estradiol (García y López, 2004).

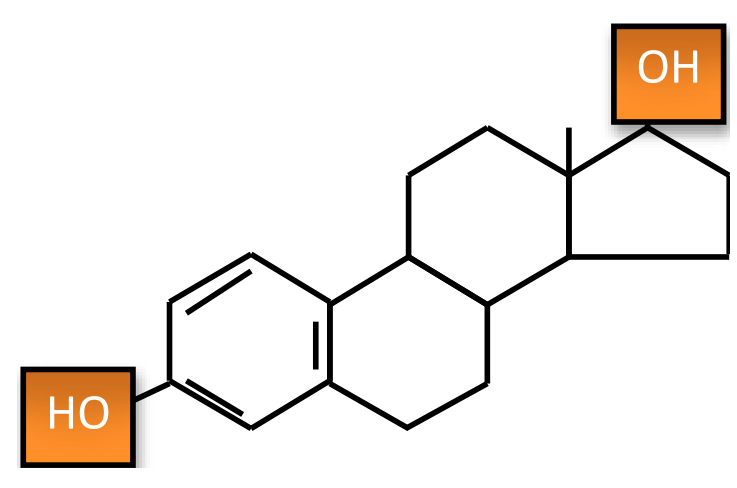

(A)<smiles>[R]Oc1ccc(C2=COc3cc(O)cc(OC4CC4)c3C2)cc1</smiles>

(C)

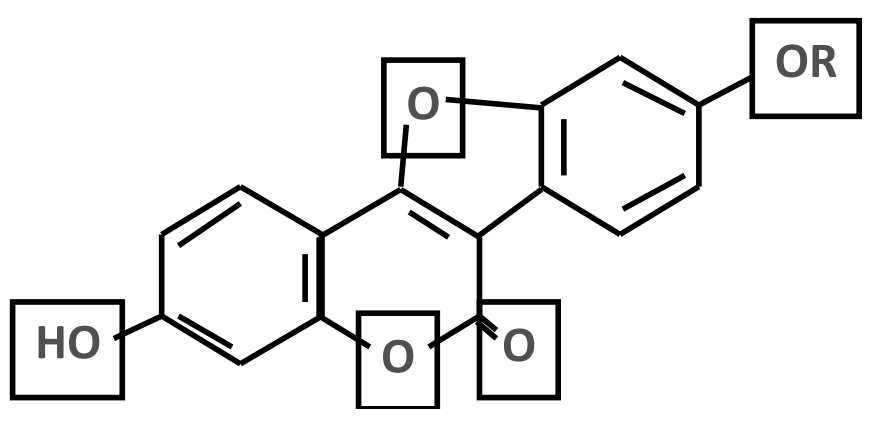

(B)

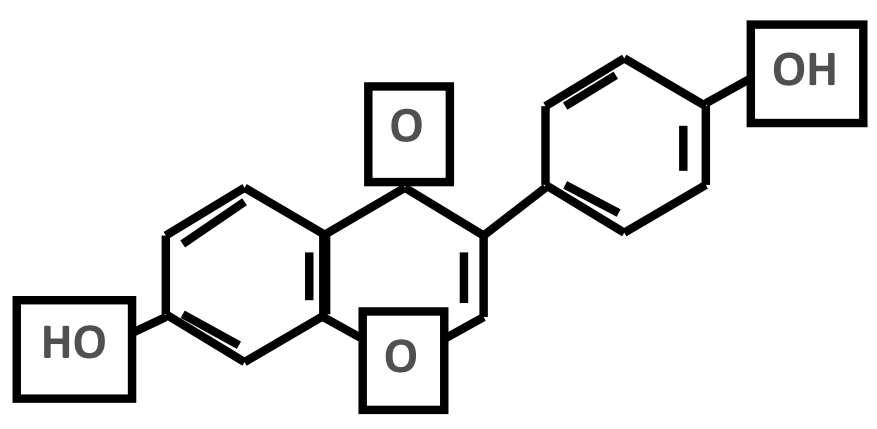

(D)

Figura 1. Estrógeno (estradiol) presente en mamíferos y los diferentes fitoestrógenos presentes en diversas fuentes vegetales. (A) 17ß-estradiol. (B) Coumestrol R=H; 7-hidroxi-12-metoxicoumestano o 4'-O-metilcoumestrol R=CH3; (C) Genisteina R=H; (D) Daidzeina.

\section{Alfalfa}

La alfalfa (Medicago sativa L.) es una leguminosa que se siembra principalmente como monocultivo y que está asociada con gramíneas debido a que a finales de otoño e invierno no crece por condiciones ambientales desfavorables. La alfalfa es cultivada en todo el mundo con propósitos alimenticios para el ganado y tiene el valor nutricional más alto de los cultivos forrajeros (SAGARPA, 2009; Esparza et al., 2009). En el caso de países como México, para el 2008 este cultivo tuvo una producción mayor a los 29 millones de toneladas, teniendo los estados de Hidalgo, Chihuahua y Guanajuato las mayores producciones con aproximadamente 5,4 y 4 millones de toneladas respectivamente. La alfalfa es comercializada tradicionalmente en pacas, verde o natural y henificada y es utilizada principalmente en el alimento del ganado lechero, aunque también es utilizado como alimento para ganado de carne, caballos, ovejas y cabras, entre otros animales, debido a su alto contenido de proteína dado que este cultivo puede fijar nitrógeno del suelo, debido a la simbiosis con la bacteria Meliloti sinorhizobium y por poseer un alto contenido de fibra digestible, la alfalfa también puede ser consumida por el ser humano en presentación de brotes, principalmente en ensaladas (tabla 3) (SAGARPA, 2009). 
Además de sus propiedades nutritivas, se han reportado propiedades antifúngicas, tónicas, diuréticas, antiinflamatorias, laxantes, hepatoprotectoras, digestivas y destoxificantes. Sus propiedades antimicrobianas han sido atribuidas al contenido de saponinas, aunque en un estudio del perfil fitoquímico y cuantificación de sus propiedades antimicrobianas de extractos en metanol y éter de petróleo de hojas de alfalfa, se encontró la presencia de lípidos, carotenoides, triterpenos, esteroles libres, alcaloides y carbohidratos mientras que en extractos metanólicos se han encontrado taninos, glicósidos y sustancias resinosas los cuales le confieren el potencial antimicrobiano contra patógenos humanos como Escherichia coli, Pseudomonas aeruginosa y Staphylococcus aureus. (Chavan et al., 2015). Por otra parte, otros estudios del perfil fitoquímico de extractos acuosos y etanol provenientes de diversas partes de la alfalfa han reportado en su composición flavonoides, glicósidos, alcaloides, saponinas y taninos, así mismo dichos extractos han mostrado actividad antimicrobiana contra microorganismos patógenos como S. aureus, E. coli, P. aerogenes, Salmonella typhimurium, Enterococcus, B. cereus, $K$. pneumoniae, y $C$. albicans. Cabe señalar que los mecanismos del efecto antimicrobiano de algunos componentes de dichos extractos, como sucede con los taninos, se deben a sus propiedades astringentes que generan la formación de complejos con enzimas y sustratos, acción sobre membranas celulares de microorganismos, y la formación de complejos con iones metálicos. Por lo anterior, se ha sugerido su uso alternativo a compuestos sintéticos antimicrobianos; sin embargo, todavía es necesario enfatizar y realizar más estudios respecto al uso terapéutico de la alfalfa (Chavan et al., 2015; Rehab, 2015).

Tabla 3. Composición de la paca verde natural o henificada de alfalfa.

\begin{tabular}{crcr}
\hline \multicolumn{2}{c}{ Verde o natural (\%) } & \multicolumn{2}{c}{ Heno (\%) } \\
\hline Agua & 77.99 & Agua & 8.50 \\
\hline Proteína bruta & 3.50 & Proteína bruta & 16.01 \\
\hline Carbohidratos & 8.43 & Carbohidratos & 40.55 \\
\hline Fibra & 6.88 & Fibra & 24.26 \\
\hline Grasa & 0.73 & Grasa & 2.73 \\
\hline Cenizas & 2.47 & Cenizas & 7.95 \\
\hline
\end{tabular}

La proteína de hoja de alfalfa ha sido reconocida por la Organización de las Naciones Unidas para la Alimentación y la Agricultura (FAO) como fuente potencial de proteína de excelente calidad para el consumo humano debido a su abundancia y valor nutritivo, siendo fuente potencial para la producción de alimentos funcionales de alta valía. Algunas propiedades fisicoquímicas y funcionales de las proteínas solubles de las hojas de alfalfa son: su temperatura de desnaturalización, capacidad de emulsificación, formación de espuma, y solubilidad, entre otras. Sin embargo, a pesar de su alto valor nutritivo, la aplicación de proteína de hoja de alfalfa en los alimentos está limitada debido a su baja solubilidad y propiedades sensoriales negativas en color, sabor y textura, siendo la hidrólisis enzimática una alternativa para eliminar estos inconvenientes. Por otra parte, los aislados hidrolizados de proteína de hoja de alfalfa han demostrado que presentan actividad antioxidante al cuantificarse esta capacidad a través de métodos in vitro. Los reportes de la capacidad antioxidante incluyen el atrapamiento del radical 1,1-difenil-2-picrilhidrazil (DPPH) con $75 \%$ a $1,6 \mathrm{mg} / \mathrm{ml}$, actividad captadora del radical hidroxil en $80 \%$ a $1,2 \mathrm{mg} / \mathrm{ml}$, actividad captadora del anión superóxido con $66,96 \%$ a $0,9 \mathrm{mg} / \mathrm{ml}$ y actividad quelante de iones metálicos con un $65,15 \%$ sobre el ion férreo a una concentración de $0,50 \mathrm{mg} / \mathrm{ml}$. Estos estudios indicarían la posibilidad de que la proteína de hoja de alfalfa tiene una potencial capacidad de reemplazo de enzimas como la superóxido dismutasa y otras enzimas antioxidantes así como nuevas aplicaciones en el desarrollo de productos alimenticios (Zhengjun et al., 2008).

La alfalfa es fuente de pequeñas cantidades de fitoestrógenos, 1-2 mg kg/MS (materia seca) como el coumestrol (figura 1) en plantas sanas. Sin embargo, cuando es afectada por enfermedades foliares ocasionadas por insectos u hongos como Pseudopeziza medicaginis genera diversos coumestanos estrogénicos, como el coumestrol, satinol y el 4' -metoxi coumestrol dando lugar a concentraciones que superan los 100 $\mathrm{mg} \mathrm{kg} / \mathrm{MS}$, lo cual es mayor a la concentración mínima de $20-50 \mathrm{mg} \mathrm{kg} / \mathrm{MS}$ considerada biológicamente activa (Muñoz et al., 2002; García y Lopez, 2004; Lenis et al., 2010; Pérez-Rivero et al., 2007) factores como la humedad y edad de la planta, disminuyen las concentraciones de coumestrol; mientras que la cantidad de fertilizante o temperatura la aumentan. El coumestrol en la alfalfa es alrededor de 30X más efectivo que 
la genisteína en ratones y se ha reportado que causa problemas relacionados con los estrógenos (endógenos) en los animales; además de que al parecer presenta un efecto acumulativo (Garcia y López, 2004).

Los diferentes fitoestrógenos tales como la genisteína, coumestrol, y lignanos presentes en leguminosas y otros vegetales pueden actuar como agonistas o antagonistas de hormonas esteroidales esto, dependiendo de la dosis que se maneje. Esta acción aparentemente contrapuesta es debida a su capacidad para unirse a los receptores estrogénicos alfa $(E R \alpha)$ del útero, glándula mamaria, sistema cardiovascular y hueso, y con mayor afinidad a receptores estrogénicos beta (ER $\beta$ ) presentes en próstata, ovarios, testículos, tracto urinario, tejido linfoide así como con el hipotálamo; Observándose la tendencia de que a medida que se incrementa la dosis de isoflavonas como genisteína y coumestrol, se generan efectos negativos como la inducción de folículos hemorrágicos, abortos, síndrome estrogénico, y supresión de picos de hormona luteinizante (LH) en hembras, mientras que en machos se altera el desarrollo testicular y decrece la cuenta de espermatozoides (Pérez-Rivero et al., 2007). Algunos otros estudios relacionados con la ingesta de fitoestrógenos y sus efectos negativos en animales experimentales mencionan que al ingerir alfalfa en concentraciones de coumestrol de 20$50 \mathrm{mg} \mathrm{kg} / \mathrm{MS}$ han dado lugar a manifestaciones estrogénicas negativas afectando específicamente el aparato reproductor del ganado lechero, en oviducto, útero, vagina y cérvix, así como un desequilibrio hormonal por la acción antigonadotrópica (inhibe la producción de FSH y LH) (Muñoz et al., 2002; Lenis et al., 2010); mientras que en los machos bovinos se ha observado que la ingestión de pasturas que contienen coumestrol, ocasiona metaplasia glandular y epitelial en próstata y glándulas bulbouretrales, además de un mayor número de espermatozoides inmaduros con disminución significativa en la movilidad (Lenis et al., 2010).

\section{CONCLUSIONES}

Los alimentos funcionales están considerados actualmente como una tendencia encaminada a la alimentación saludable debido a los malos hábitos alimentarios derivados del modo de vida contemporáneo. Así mismo, la tendencia a su consumo sigue abarcando cada vez más a diversos países alrededor del mundo incluidos los de América Latina, contribuyendo así a la integración social y cuidado de la salud con respecto de la alimentación. Actualmente son variadas las líneas de investigación y desarrollo en el área, las cuales pueden dar valor agregado a las materias primas las cuales son abundantes en países latinoamericanos (Illanes, 2015). Las alimentos de origen vegetal muestran una variedad de compuestos biológicamente activos que les confieren un beneficio adicional al consumidor más allá del nutricional; un ejemplo de ello son las leguminosas las cuales contienen fitoestrógenos, compuestos con actividad estrogénica y a la que deben su nombre, por lo que les ha llevado a ser considerados para su aplicación en terapias de reemplazo hormonal en síntomas asociados a la menopausia y osteoporosis en mujeres en el climaterio. Además, su consumo ha sido relacionado con efectos benéficos contra diferentes enfermedades como algunos tipos de cáncer, obesidad, diabetes, y enfermedades cardiovasculares, entre otras.

Por otra parte, estudios realizados por diversos investigadores en animales han reportado como resultado efectos adversos debido al consumo de este tipo de compuestos específicamente en el aparato reproductivo. Los fitoestrógenos pueden actuar como agonistas o antagonistas de hormonas esteroidales, aunque este efecto está relacionado con la dosis que se maneje. Si bien se encuentran documentados los efectos que tienen estos fitoquímicos en animales experimentales los cuales pueden dar lugar a daños económicos debido a los cambios reproductivos, esto demuestra que aún falta mucha más información y estudios que realizar respecto al resultado que tienen los fitoestrógenos en el desarrollo y función reproductiva de los animales, el agonismo y antagonismo de su efecto así como la concordancia en el riesgo-beneficio de su consumo.

\section{REFERENCIAS}

Abreu, A. (2012). Prebióticos, probióticos y simbióticos. Revista de Gastroenterología de México, 77, 26-28.

Alvídrez, A., González, B. E. \& Jiménez, Z. (2002). Tendencias en la producción de alimentos: alimentos funcionales. RESPYN, 3(3). 
Araya, H. \& Lutz, M. (2003). Alimentos funcionales y saludables. Revista chilena de nutrición, 30(1), 8-14.

Ávalos, A. \& Pérez, E. (2009). Metabolismo secundario de plantas. REDUCA, 2(3), 119-145.

Badui, S. (2015). Inocuidad en la industria alimentaria. Industria Alimentaria, 37(3), 14-26.

Bedascarrasbure, E., Maldonado, L., Álvarez, A. \& Rodríguez, E. (2004). Contenido de fenoles y flavonoides del propoleos argentino. Acta Farmacéutica Bonaerense, 23(3), 369-372.

Bolet, M. \& Socarrás, M. (2010). Alimentación adecuada para mejorar la salud y evitar enfermedades crónicas. Revista Cubana de Medicina General Integral, 26(2), 321-329.

Bonilla, C. (2004). Isoflavonas en ginecología, terapia no convencional. Revista Colombiana de Obstetricia y Ginecología, 55(3), 209-217.

Carrasco, C. A., \& Guerra, M. (2010). Lactosuero como fuente de péptidos bioactivos. Anales Venezolanos de Nutrición (23)1, 42-49.

Cendales, L. V. R., \& Silva, H. J. D. (2011). Phytoestrogens: an alternative to hormone replacement therapy. Duazary Revista Facultad de Ciencias de la SaIud. Suplemento 2011. Diciembre pp. 84-89. http:// www.researchgate.net/profile/Carmen_Rosario_ Bocanegra_Amaya/publication/235725645_Article_VPH/links/Ofcfd512e89f3e70bd000000.pdf\#page $=84$

Chasquibol, N., Lengua, L., Delmás, I., Rivera, D., Bazán, D., Aguirre, R. \& Bravo, M. (2003). Alimentos funcionales o fitoquímicos, clasificación e importancia. Revista Peruana de Química e Ingeniería Química, 5(2), 9-20.

Chavan, S. S., Jadhav, R. S., Khemnar, K. S. \& Tambe, V. B. (2015). Evaluation of Antibacterial Activity and Phytochemical Screening of Medicago sativa Leaves. International Journal of Current Research and Academic Review, 3(5), 308-313.

Cortés, M., Chiralt, A. \& Puente, L. (2005).Alimentos funcionales: una historia con mucho presente y futuro. Vitae, 12, 5-14.
Cortés, M., García, A., \& Suárez, H. (2007). Fortification of edible mushrooms (Pleurotusostreatus) with calcium, selenium and vitamin C. Vitae, Revista de la Facultad de Química Farmacéutica, 14(1), 16-24.

Domínguez , K.N. , Cruz , A. E. , González , H., Gómez , L. C., García , M. \& Rodríguez , G. M. (2014). El efecto antihipertensivo de las leches fermentadas. Revista Argentina de Microbiología, 46(1), 58-65.

Esparza, J., Garay, A., Pérez, J., Haro, J., Gallardo, F., Hernández, P., González, S. \& Carrillo, A. (2009). Análisis de crecimiento estacional de una pradera asociada alfalfa-pasto ovillo. Técnica pecuaria en México, 47(2), 173-188.

García, D. \& López O. (2004). Los fitoestrógenos: ¿mito o amenaza para la alimentación animal en el trópico? Pastos y Forrajes, 27(4), 303-316.

Garrido, A., De la Maza, C. \& Valladares, L. (2003). Fitoestrógenos dietarios y sus potenciales beneficios en la salud del adulto humano. Revista médica de Chile, 13(11), 1321-1328.

Hernández, E. \& Verdalet, I. (2003). Revisión: alimentos e ingredientes funcionales derivados de la leche. Archivos Latinoamericanos de Nutrición, 53(4), 333-347.

Illanes, A. (2015). Alimentos funcionales y biotecnología. Revista Colombiana de Biotecnología, 17(1), 5-8.

Jiménez, F. (2013). Emulsiones múltiples: compuestos bioactivos y alimentos funcionales. Nutrición Hospitalaria, 28(5), 1413-1421.

Lenis, Y., Gutiérrez, T. \& Tarazona, A. (2010). Efectos de los fitoestrógenos en la reproducción animal. Revista Facultad Nacional de Agronomía Medellín, 63(2), 5555-5565.

Lorente, B. F. \& Serra, D. J. (2001). Alimentos funcionales: probióticos. Acta Pediatr Espan, 59(3), 150-5.

Lutz, M. (2009). III. Alimentos funcionales en la prevención de enfermedades crónicas no transmisibles. Aspectos nutricionales y saludables de los productos de panificación, 38. http://www.agro. unc.edu.ar/ uninvestigacion/cyted/files/2009_ 
Aspectos_nutricionales_y_saludables_de_los_ productos_de_panificacion.pdf\#page $=37$

Madrigal, L. \& Sangronis, E. (2007). La inulina y derivados como ingredientes claves en alimentos funcionales. Archivos Latinoamericanos de Nutrición, 57(4), 387-396.

Martínez, I. \& Villezca, P. (2005). La alimentación en México, un estudio a partir de la encuesta nacional de ingresos y gastos de los hogares y de las hojas de balance alimenticio de la FAO. Ciencia UANL, 8(2), 196-208. http://eprints.uanl.mx/1659/1/art_ de_la_fao.pdf Base de datos

Maydata, B. (2002). Chocolate, polifenoles y protección a la salud. Acta Farm. Bonaerense, 21(2), 149-52.

Melo-Ruiz, V. \& Cuamatzi-Tapia, O. (2006). Bioquímica de los procesos metabólicos. Reverte. pp. 364.

Muñoz, R., Murillo, A., Pérez, J. \& Córdova, A. (2002). Parámetros reproductivos en vacas holstein alimentadas con alfalfa alta en cumestrol. Archivos de Zootecnia, 51(195), 373-376.

Mohamed R, El-Desoukey. A. (2015). Phytochemical and Antimicrobial Activity of Medicago sativa (Alfalfa) as Source of Animal Food Against Some Animal Pathogens. Global Veterinaria, 14(1), 136-141.

Murcia, J.L. (2013). Alimentos funcionales. Un mercado al alza que mueve en el mundo cerca de 100.000 millones de euros anuales. Distribución y Consumo 49, 5. http://www.mercasa.es/files/ multimedios/1387540447_Alimentos_funcionales_48-50.pdf.

Olagnero, G., Abad, A., Bendersky, S., Genevois, C., Granzella, L. \& Montonati, M. (2007). Alimentos funcionales: fibra, prebióticos, probióticos y simbióticos. Diaeta, 25(121), 20-33.

OMS. (Octubre, 2009). 10 datos sobre la inocuidad de los alimentos. Disponible en: http://www.who.int/ features/factfiles/food_safety/es/, accesado el 21 de febrero de 2016.

Ortega Anta, R.M., Jiménez Ortega A. I., \& Tur Mari J.A. (2010). Alimentos funcionales y salud. In: Alimentos funcionales y salud en etapas infantil y ju- venil. J. Aranceta \& A. Gil (coord). Editorial Médica Panamericana. pp. 203.

Ortiz Nievas, V.T. (2010). Cuidando La salud con los fitoestrógenos. Revista UNIMAR, 28, 19-21.

Pérez-Rivero, J. J., Aguilar-Setién, A., Martínez-Maya, J. J., Pérez-Martínez, M., \& Serrano, H. (2007). Los fitoestrógenos y el efecto de su consumo en diferentes órganos y sistemas de animales domésticos. Agricultura Técnica, 67(3), 325-331.

Pérez-Urria Carril, E., \& Ávalos García, A. (2009). Metabolismo secundario de plantas. Reduca, 2(3), 119-145.

Ramos, L. y Díaz H. (2011). Fitoestrogenos: una alternativa en terapia de reemplazo hormonal. Duazary Revista Facultad de Ciencias de la Salud, 8(spl) 84-89.

Rodríguez, M., Monereo, S. \& Begoña, B. (2003). Alimentos funcionales y nutrición óptima: ¿Cerca o lejos? Revista Española de Salud Pública, 77(3), 317-331.

Rubiano, L. (2006). Alimentos funcionales, una nueva alternativa de alimentación. Orinoquia, 10(1): 16-23.

SAGARPA/ProMercado. (2009) Diseño de Estrategias de Mercado, Logísticas y de Adecuación de Productos para la Integración de la Alfalfa Mexicana en el Comercio Global de Forrajes. Disponible en : http://www.sagarpa.gob.mx/agronegocios/Documents/Estudios_promercado/ALFALFA.pdf, accesado el 02 de octubre de 2008

Sastre, A. (2003). Fibra y prebióticos: conceptos y perspectivas. Gastroenterol Hepatol, 26, 6-12.

Serrano, M.E.D., López, M.L., \& Espuñes, T.D.R.S. (2006). Componentes bioactivos de alimentos funcionales de origen vegetal. Revista Mexicana de Ciencias Farmacéuticas, 37(4), 58-68.

Shemesh, M., Lindner, H. \& Ayalon, N. (1972). Affinity of rabbit uterine oestradiol receptor for phyto-oestrogens and its use in a competitive protein-binding radioassay for plasma coumestrol. Journal of Reproduction and Fertility, 29, 1-9. 
Suárez, J. (2013). Microbiota autóctona, probióticos y prebióticos. Nutrición Hospitalaria 28, 38-41.

Thompson, L., Boucher, B., Liu, Z., Cotterchio, M. y Kreiger, N. (2006). Phytoestrogen content of foods consumed in Canada, including isoflavones, lignans, and coumestan. Nutrition and Cancer, 54(2), 184-201.

Tomás-Barberán F. A. (2003). Los polifenoles de los alimentos y la salud. Alim. Nutri. Salud, 10(2), 41-53.

Toxqui, L., De Piero, A., Courtois, V., Bastida, S., Sánchez, F. \& Vaquero, M. (2010). Deficiencia y sobrecarga de hierro; implicaciones en el estado oxidativo y la salud cardiovascular. Nutrición Hospitalaria, 25(3), 350-365.
Toyama, G. (2010). Prebióticos, probióticos y simbióticos en la dietoterapia de los pacientes quemados. DIAETA, 28, 7-14.

Varela Moreiras, G. (2010). Alimentos funcionales. In: Alimentos funcionales y salud en etapas infantil y juvenil. J. Aranceta y A. Gil (coord). Editorial Médica Panamericana. pp. 203.

Zhengjun, X., Junrong, H., Xueming, X. \& Zhengyu, J. (2008). Antioxidant activity of peptides isolated from alfalfa leaf protein hydrolysate. Food Chemistry $111,370-376$. 